\title{
Comparison of Papanicolaou and Acridine Orange stains in the Diagnosis of Trichomonas Vaginalis Infection in Vaginal Discharge
}

\author{
Neena Piyush Doshi \\ Dept of Pathology, GMERS Medical College, Gotri, Vadodara (India)
}

\begin{abstract}
Background: Trichomonas vaginalis is the most common cause of vaginitis. Diagnosis based on clinical presentation is often inaccurate. Clinical presentation with adjuvant laboratory evidence of trichomoniasis is required for the confirmation of the diagnosis and initiation of therapy. The aim of the present study was to compare the sensitivity and specificity of rapid staining Acridine Orange method for detection of trichomonas vaginalis infection with the routinely employed Papanicolaou staining.

Materials and Methods: Vaginal swab smears were prepared from 300 patients attending gynaecology outpatients' clinic of S.S.G. Hospital, Vadodara with complain of abnormal vaginal discharge. The prospective study was conducted over a period of two years (from January' 98 to December' 99). The Papanicolaou and Acridine Orange stained smears were screened for presence of Trichomonas vaginalis infection.

Results: In the present study, the incidence of Trichomonas vaginalis infection was $12.3 \%$. Considering Papanicolaou stain as standard, the sensitivity and specificity of Acridine Orange (AO) stain for detection of T. vaginalis was $92.8 \%$ and $93.01 \%$ respectively. The positive and negative predictive values were $74.2 \%$ and $99.2 \%$, while false positive and false negative values are $3 \%$ and $0.66 \%$ respectively.

Conclusion: Trichomonas vaginalis infection is a sexually transmitted disease of the reproductive age group, which needs to be timely diagnosed to prevent its adverse effects. The rapidity, ease and reliability of Acridine Orange stain justify its use in routine laboratory diagnosis of Trichomonas vaginalis infection.
\end{abstract}

\section{Keywords: Richomonas Vaginalis, Papanicolaou Stain, Acridine Orange Stain, Fluorescence}

\section{Introduction}

Trichomonas vaginalis is the most common sexually transmitted disease (STD) worldwide and affects an estimated $170-180$ or more million people annually. ${ }^{[1,2]}$ The WHO estimates an incidence of 276 million new cases each year. ${ }^{[3]}$ Trichomoniasis is almost entirely a disease of the childbearing era. There is no doubt that this infection is sexually transmitted. Nevertheless, the male carrier is generally symptom free, because a certain amount of oestrogenic activity is required for the trichomonads to reach full maturity. In some instances, the infection can be acquired by inadequate hygiene or the use of infected towels, toilet seats, douche nozzles, clothes, bed linen and contaminated swimming pool water ${ }^{[1]}$ Iatrogenic spread occurs through use of contaminated gloves and improperly sterilized surgical instruments especially vaginal speculum. Reinfection can occur from untreated sexual partner, extravaginal site lodgment of parasite, which is inaccessible to local therapy and, urinary tract.

Incubation period of Trichomonas vaginalis (T. vaginalis) varies from 5 to 28 days. ${ }^{[4]} 75 \%$ patients complain of profuse yellowish-white, greenish-yellow to creamy-white vaginal discharge. It is usually thin, but up to $30 \%$ cases have thick discharge. $7-10 \%$ patients complain of foul smelling discharge. Pathognomonically the discharge is foamy or frothy due to fermentation of carbohydrates, which is seen in about only $10 \%$ of patients. ${ }^{[5,6]}$ The presence of secondary infection may mask the self-diagnostic sign of frothy discharge. Burning and irritation of vulva becomes worse after periods due to increased levels of oestrogen, which favors T. vaginalis growth. Infection may be associated with genital pruritus and pain (dyspareunia, dysmenorrhea, lower abdominal pain and lower backache). It may cause urinary symptoms like dysuria, frequency and/or urgency.

Since the introduction of metronidazole for the treatment of trichomoniasis, a major problem in the control of this disease has been the accuracy of diagnosis. Also number of strains resistant to metronidazole is on an increase. ${ }^{[5]}$ And there is no alternative treatment currently. ${ }^{[3]}$ It is so widespread and causes so much ill health among young women, that it has become an important social problem. In addition to abnormal vaginal discharge, it is increasingly associated with endometritis during pregnancy, pre-term labor, premature rupture of membranes, abortion, post- 
cessarean infection, and human immunodeficiency virus (HIV) infection. ${ }^{[4,7]} \mathrm{T}$. vaginalis is associated with 1.52fold increased risk of HIV-1 acquisition. ${ }^{[8]}$ Therefore, the detection of T. vaginalis infection is of utmost importance. The elimination of trichomoniasis depends not only on treatment of the symptomatic cases, but also in detecting carrier patients who have mild symptoms or none, or who accept their vaginal discharge as 'normal' because every woman has a discharge anyway.

Wet smear examination though simple is an insensitive method. A minimum concentration of $10^{4}$ organisms per milliliter of vaginal discharge is necessary for wet mount to be positive. ${ }^{[27}$ The acknowledged insensitivity is in partly due to the rapid loss of the characteristic motility of the microorganism. A large number of culture and staining techniques have been designed to increase the reliability of laboratory diagnosis of T. vaginalis. Diamond's medium is considered the gold standard. More recently culture method composed of liquid medium is used..$^{[5]}$ Culture is a sensitive method for detecting trichomonas as compared to wet smear preparation, however it is relatively slower, requiring 2-7 days for diagnosis. ${ }^{[4]}$

Papnicolaou stain, Giemsa stain, and other Romansowsky stains are amongst the stains for vaginal smears commonly used for the diagnosis of $\mathrm{T}$. vaginalis. ${ }^{[2]}$ It is difficult to diagnose when trichomonads are scanty. Rounded forms of trichomonads can be confused easily with polymorphonuclear leukocytes or may look like dysplastic cells. Bare nuclei, degenerated cells and inspissated mucous plugs can be confused with trichomonads. The need for more sensitive, specific rapid and simpler method for detecting trichomonas vaginalis infection, to overcome the drawbacks of the existing methods, was recognized for a long time. More recent reports, describe the use of Acridine Orange stain using fluorescence microscope. The stain is differently adsorbed by the parasite and holds promise in improving the diagnostic ability of trichomonas vaginalis infection.

The present study is undertaken to evaluate the usefulness of Acridine Orange staining in the diagnosis of vaginal trichomoniasis and compare the efficacy with Papanicolaou staining routinely employed on vaginal smears. To define the performance characteristic of Acridine Orange stain, Papanicolaou stain is considered as standard for the present study.

\section{Materials and Methods}

The present study was carried out in the Department of Pathology, Medical College and S.S.G. Hospital, Vadodara,
Gujarat, India over a period of two years (from January' 98 to December' 99). 300 patients attending gynaecology clinic of S.S.G. Hospital with complain of abnormal vaginal discharge formed the 'target group' of this prospective study. A detailed clinical history was taken. The findings were noted down in the performa.

Smear Collection: Trichomonas vaginalis infection is essentially of the vaginal epithelium. The parasites shelter between rugae and acquire their food from glycogen rich vaginal epithelium. The specimen for the present study was thus collected from the posterior vaginal fornix pool. The vaginal swab containing the specimen was rolled onto two clean slides, one for Papanicolaou stain and other for Acridine Orange. The slides were immediately fixed by immersing in a coplin jar full of cytofix (a mixture of equal volumes of $95 \%$ ethanol and ether).

Processing of Smears: The smears were fixed in cytofix for a minimum of 30 minutes and stained by Papanicolaou and Acridine Orange. In case of any delay in staining, the slides are removed from cytofix and air-dried. For Acridine Orange stain, the staining characteristics are better with cytofixed slides than air-dried smears.

\section{Papanicolaou (Pap) Staining Method:}

$\begin{array}{ll}90 \% \text { ethyl alcohol } & 10 \text { dips } \\ 70 \% \text { ethyl alcohol } & 10 \text { dips } \\ 50 \% \text { ethyl alcohol } & 10 \text { dips } \\ \text { Running water } & 1 \text { minute } \\ \text { Harris's haematoxylin } & 2 \text { minutes } \\ \text { Running water } & 2 \text { minutes } \\ \text { Acid alcohol } & 1 \text { dip } \\ \text { Running water } & 5 \text { minutes } \\ 50 \% \text { ethyl alcohol } & 4 \text { dips } \\ 70 \% \text { ethyl alcohol } & 4 \text { dips } \\ 90 \% \text { ethyl alcohol } & 4 \text { dips } \\ \text { Orange G. 6 (O.G. 6) } & 5 \text { minutes } \\ 95 \% \text { ethyl alcohol } & 4 \text { dips } \\ 95 \% \text { ethyl alcohol } & 4 \text { dips } \\ \text { Eosin azure 50 (EA 50) } & 2 \text { minutes } \\ 95 \% \text { ethyl alcohol } & 4 \text { dips } \\ 100 \% \text { ethyl alcohol } & 4 \text { dips } \\ 100 \% \text { ethyl alcohol } & 4 \text { dips } \\ \text { Xylol } & \text { half an hour } \\ \text { Xylol } & \text { half an hour } \\ \text { Blotted and mounted with Distrene } 80 \text { Dibutylphthalate } \\ \text { Xylene (DPX) }\end{array}$

Microscopic Appearance of T. Vaginalis Infection in Pap Smear: In Papanicolaou smears, the parasites appear as gray-green, round or elliptical structures, varying in size 
from 8 to $20 \mu \mathrm{m}$ (Fig. 1). Large size trichomonads appear when growing conditions are unfavorable while smaller sizes prevail in favorable growing conditions.

The smears from trichomoniasis are usually rich in leukocytes. There is excessive cytolysis. Debris and large plaques of necrotic cells are common. These make the background appear 'dirty'. Clustering of leukocytes and macrophages around epithelial cells, called the 'cannon ball or BB shot appearance', may be observed. There is excessive eosinophilia involving the normally basophilic cells such as the intermediate and the parabasal (Fig. 2). Leptothrix, which are long filamentous bacterial structures, commonly occur in conjunction with trichomonads. All the above features are clue to the diagnosis of trichomonas vaginalis infection, however identification of the parasite is essential before the diagnosis can be established with assurance.

It may be difficult to find $\mathrm{T}$. vaginalis in severe inflammation (Fig. 3). Inspissated material (Fig. 4) and bare nuclei (Fig.5) may be confused with T. vaginalis.

\section{Acridine Orange (AO) Staining Method:}

$\begin{array}{ll}80 \% \text { ethyl alcohol } & 4 \text { dips } \\ 70 \% \text { ethyl alcohol } & 4 \text { dips } \\ 50 \% \text { ethyl alcohol } & 4 \text { dips } \\ \text { Distilled water } & 4 \text { dips } \\ 1 \% \text { acetic acid } & 4 \text { dips } \\ \text { Distilled water } & 1 \text { dip } \\ \text { Working solution of AO }(0.01 \%) & 3 \text { minutes } \\ \text { Phosphate buffer }(\mathrm{pH} 6.4) & 1 \text { minute } \\ \text { M/10 Calcium chloride } & 30 \text { seconds } \\ \text { Phosphate buffer }(\mathrm{pH} 6.4) & \text { wash } \\ \text { M/10 Calcium chloride } & 30 \text { seconds } \\ \text { Phosphate buffer }(\mathrm{pH} \mathrm{6.4)} & \text { dip }\end{array}$

Smears were allowed to dry. Stained slides keep no longer than 24 hours at room temperature and 2 months at $4^{\circ} \mathrm{C}$. The stock solution does not keep for more than 6 months.
Microscopic Appearance of $\mathbf{T}$. Vaginalis Infection Using AO Fluorescence Dye: Prior to screening, the AO stained smears are mounted in buffer. The exciter filter of the fluorescence microscope was adjusted to $400 \eta \mathrm{m}$ and barrier filter was used as a combination of filters $44(10 \%$ transmission at $440 \eta \mathrm{m})$ and $53(10 \%$ transmission at $530 \mathrm{\eta m})$. Acridine Orange is a nucleic acid stain, staining differently DNA and RNA, the former fluoresces yellow to green and the latter bright red under ultraviolet illumination. Acridine Orange is very sensitive for picking up organisms. Trichomonas vaginalis trophozoites stain a characteristic brick red with a yellowish-green banana shaped or rounder nucleus (Fig. 6). The cytoplasm of leukocytes does not stain but the nuclei fluoresce bright green. Yeasts stain red. Bacteria including those within phagocytic cells stain bright red. ${ }^{[2]}$ The likelihood that the additional positives detected by Acridine Orange stain would not be so, is slight, since nothing else in smears significantly resembles trichomonads to cause difficulty.

\section{Results}

In the present study, comparing results by both methods in each case, 26 cases were positive for T. vaginalis infection by both methods. There was disparity in 11 cases, in which 2 cases were positive by Papanicolaou stain and not by Acridine Orange, while 9 cases were positive by Acridine Orange but negative by Papanicolaou stain. Combining both, there were 37 positive cases in all as shown in table 1 . Incidence of Trichomonas vaginalis infection was $12.3 \%$.

Considering Papanicolaou stain as the standard for present study, the sensitivity and specificity of Acridine Orange (AO) stain for detection of $\mathrm{T}$. vaginalis is calculated as $92.8 \%$ and $93.01 \%$ respectively. Positive and negative predictive values are $74.2 \%$ and $99.2 \%$ respectively, while false positive and false negative values are $3 \%$ and $0.66 \%$ respectively.

Table 1: Pap and AO stained smears positive for T. vaginalis infection.

\begin{tabular}{|l|c|c|c|c|}
\hline \multirow{2}{*}{} & \multirow{2}{*}{} & \multicolumn{2}{|c|}{ Papanicolaou stain } & \multirow{2}{*}{ Negative } \\
\cline { 2 - 5 } & & Positive & $\mathrm{b}=09$ & 35 \\
\hline \multirow{2}{*}{ Acridine Orange } & P o s it i v & $\mathrm{a}=26$ & $\mathrm{~d}=263$ & 265 \\
\cline { 2 - 5 } & N e g a t i ve & $\mathrm{c}=02$ & 272 & Total=300 \\
\hline
\end{tabular}

$a=$ true positive, $b=$ false positive, $c=$ false negative, $d=$ true negative 
Table 2: Incidence of Trichomonas vaginalis in female vaginal discharge as observed in different studies.

\begin{tabular}{|c|c|c|}
\hline Sr. No. & Work done by & Incidence (\%) \\
\hline 1. & Karaman et al $^{[0]}$ & 8.1 \\
\hline 2. & Klinger EV et al[10] $^{[10]}$ & 10.7 \\
\hline 3. & Rassjo EB et al[1]] & 8.0 \\
\hline 4. & Menezes CB et a[ $\left[{ }^{[1]}\right.$ & 15 \\
\hline 5. & Khamees SS & 26.8 \\
\hline 6. & Present study & 12.3 \\
\hline
\end{tabular}

Table 3: Sensitivity of Acridine Orange stain for detecting T. vaginalis infection.

\begin{tabular}{|c|c|c|}
\hline Sr. No. & Work done by & Sensitivity (\%) \\
\hline 1. & Khatoon R et al ${ }^{[2]}$ & 100 \\
\hline 2. & Radonjic IV et al ${ }^{[14]}$ & 71.43 \\
\hline 3. & Hussein AH et al ${ }^{[15]}$ & 81.8 \\
\hline 4. & Present study & 92.8 \\
\hline
\end{tabular}

Table 4: Specificity of Acridine Orange stain for detecting T. vaginalis infection.

\begin{tabular}{|c|c|c|}
\hline Sr. No. & Work done by & \multicolumn{1}{c|}{ Specificity (\%) } \\
\hline 1. & Khatoon R et al $^{[2]}$ & 98 \\
\hline 2. & Radonjic IV et al ${ }^{[14]}$ & 99.44 \\
\hline 3. & Hussein AH et al[ ${ }^{[15]}$ & 100 \\
\hline 4. & Present study & 93.01 \\
\hline
\end{tabular}

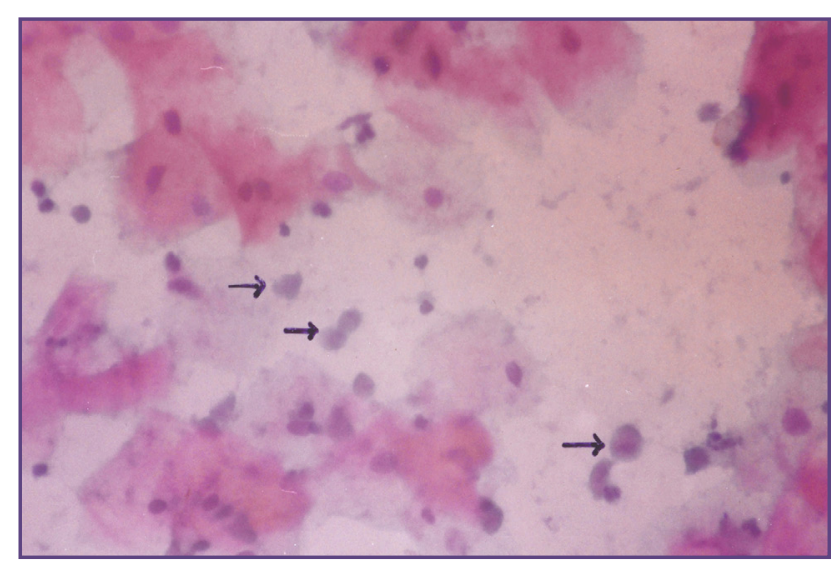

Fig. 1: (Pap stained vaginal smear, 40x view): Trichomonas vaginalis in Papanicolaou stained vaginal smears.

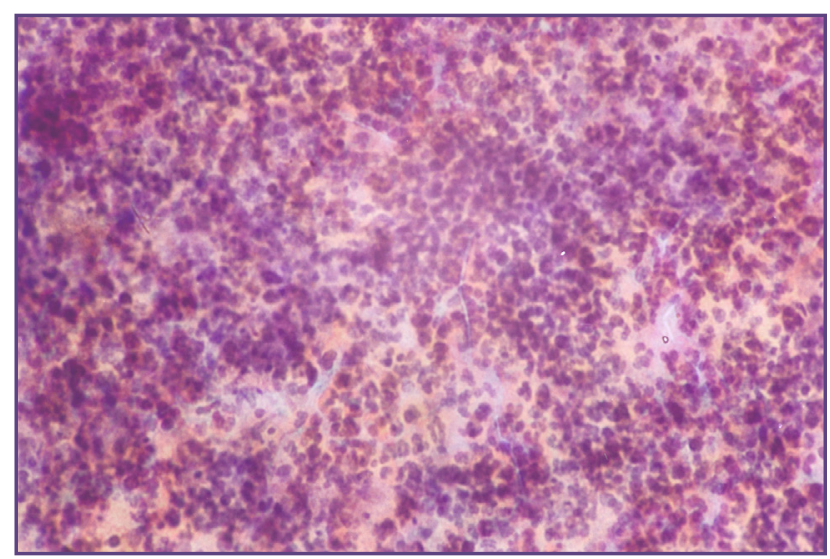

Fig. 3; (Pap stained vaginal smear, 40x view): Severe inflammation making it difficult to find the trichomonads.

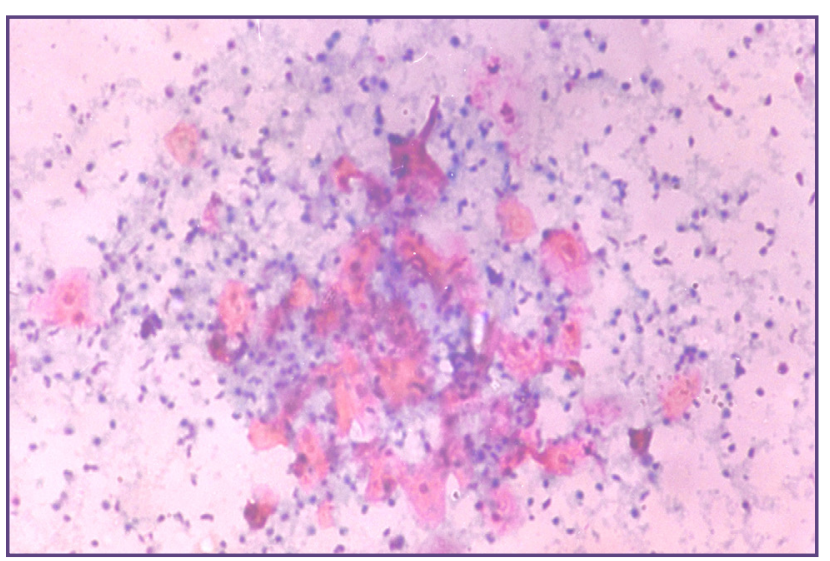

Fig. 2; (Pap stained vaginal smear, 10x view): Cannon ball appearance, dirty background and excess eosinophilia.

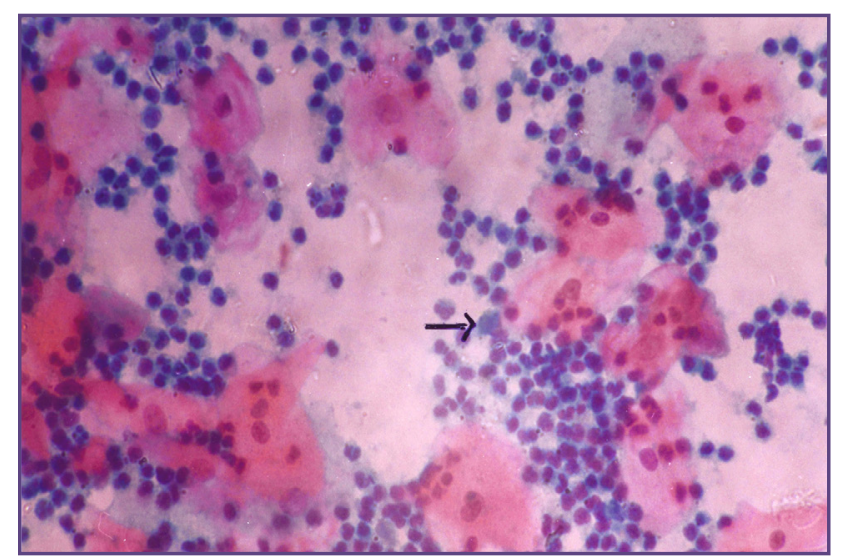

Fig. 4: (Pap stained vaginal smear, 40x view): Inspissated material can be confused with $T$. vaginalis. 


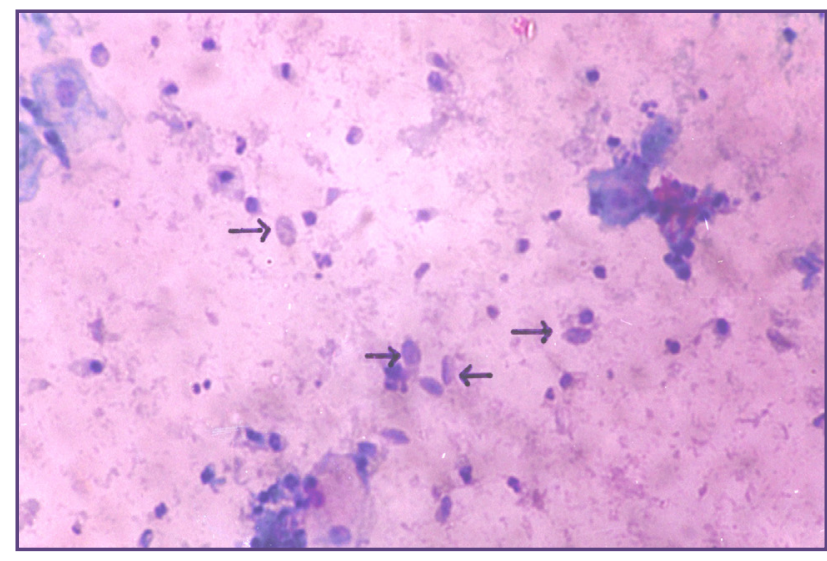

Fig. 5: (Pap stained vaginal smear, 40x view): Bare nuclei can be confused with $T$. vaginalis.

\section{Discussion}

Long considered a minor sexually transmitted disease (STD) with few associated complications, T. vaginalis is now known to be associated with pregnancy related complications and morbidity in non-pregnant women. Besides, trichomoniasis can be asymptomatic in nearly $50 \%$ of the infected women or they may present with wide variety of symptoms. Once established, the infection may persist for long periods in women. ${ }^{[6]}$

As per the published literature, the prevalence of $\mathrm{T}$. vaginalis ranges from $0.4-27.4 \%$ in women. ${ }^{[4]}$ In India, trichomoniasis accounts for $2-7 \%$ of all sexually transmitted infections. ${ }^{[6]}$ In the present study, Acridine Orange stained smears picked up $11.6 \%$ positive cases while Papanicolaou stain picked up 9.3\% positive cases. Combining both results there were 37 positive cases in all, thus an incidence of $12.3 \%$. Table 2 shows the incidence of Trichomonas vaginalis infection as observed by different workers.

The sensitivity of Acridine Orange stain in detecting Trichomonas vaginalis infection as observed by different workers is put in table 3 . The sensitivity of Acridine Orange stain as observed by different workers varied between 33$93.8 \%$. The wide range of sensitivity could be attributed to sampling error, faulty staining technique and mild infection. Nassef NE et al showed $86.7 \%$ sensitivity by AO stain as compared to culture. ${ }^{[7]}$ The specificity of Acridine Orange stain in detection of Trichomonas vaginalis as observed by workers is put in table 4 .

Accurate diagnosis of $\mathrm{T}$. vaginalis, whether symptomatic or asymptomatic, is affected by many variables like patient factors, clinician's experience, specimen sampling and processing and expertise of those who perform microscopy. Traditional wet mount smear is rapid method

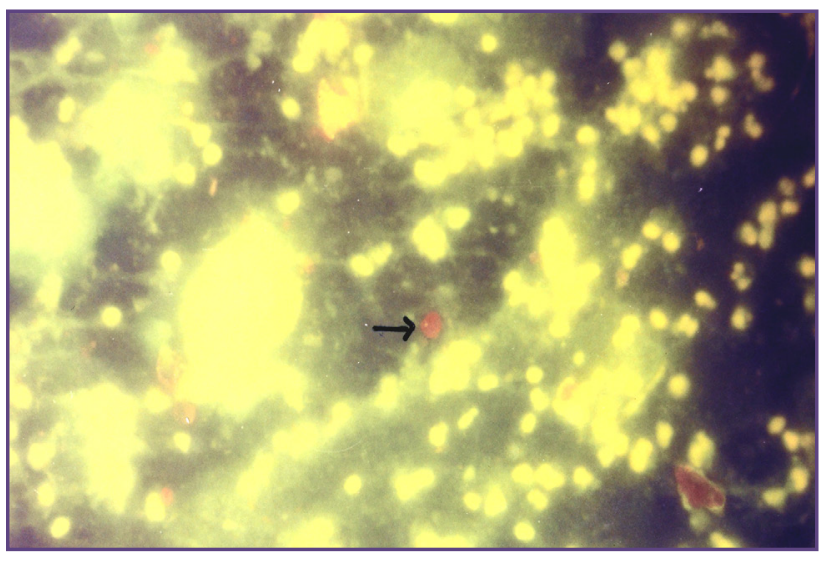

Fig. 6; (A0 stained vaginal smear, 40x view): T. vaginalis in Acridine Orange stained smears.

but with low sensitivity and specificity. Besides it cannot be performed in clinics with heavy patient load. Cultures considered as gold standard are relatively slow. In order to identify $\mathrm{T}$. vaginalis by culture, it is necessary to have an inoculum of 300-500 trichomonas/ml. ${ }^{[14]}$ Giemsa stain is not appropriated since it stains only the nucleus of the organism. $^{[12]}$ Papanicolaou stained smear examination needs competence and expertise. False-positives as well as false negatives are common with this technique. ${ }^{[3]}$ Cellular picture of inflammatory vaginal discharge is similar particularly in trichomonas and Chlamydia. Acridine orange staining has the advantage of ease and rapidity to perform with higher sensitivity and specificity for diagnosis of Trichomonas vaginalis infection. The only disadvantage is the requirement of special microscope and availability of the dye. However, the cost of fluorescent microscope has decreased, so simplicity and speed of AO staining and diagnosis should encourage its' use. ${ }^{[14]}$ AO stained smears lose their fluorescence; hence it is not possible to have permanent record. Latex agglutination, enzyme-linked immunosorbent assay, immunochromatography-based tests, direct immunofluorescence assay, DNA hybridization probe test and polymerase chain reaction (PCR) are the newer tests. ${ }^{[14,7,3]}$

\section{Conclusion}

High prevalence, increasing resistance to treatment and association with health complications are the burning issues related to $T$. vaginalis infection. A definitive diagnosis of trichomonas vaginalis infection cannot be made on clinical symptoms alone. Diagnosis must be laboratorial. Considering the higher sensitivity, specificity and the rapidity and ease of Acridine Orange staining, its use in routine laboratory diagnosis of trichomoniasis is justifiable. This will provide early diagnosis and prompt treatment to patients suffering from the infection. It will 
help in reducing disease associated morbidity and adverse health outcomes. Although, in gynaecologic practice the value of Papanicolaou stained smears cannot be under estimated because of its primary usefulness in screening women for cervical cancer, but Acridine Orange staining is advocated in cases suspected of trichomonas infection.

\section{Acknowledgements}

I express my gratitude to Dr. M.R. Desai, then Professor \& Head, O/G, Medical College, Vadodara for allowing me to collect cytology smears. I sincerely thank Dr. R.Z. Patel and Dr. R.K. Pasle, Pathologists, Vadodara in helping me through the making of stains. The work

wouldn't have been possible without the help of Dr. Rajapurkar, MD, MPUH, Nadiad, who very kindly allowed me to avail the facility of the institutional fluorescence microscope.

\section{References}

1. Pereira-Neves A, Benchimol M. Trichomonas vaginalis: in vitro survival in swimming pool water samples. Exp Parasitol. 2008 Mar,118(3):438-41.

2. Khatoon R, Jahan N, Khan HM, Rabbani T, Ahmad S. Evaluation of Different Staining Techniques in the Diagnosis of Trichomonas vaginalis Infection in Females of Reproductive Age Group. J Clin Diagn Res.. 2014 Dec;8(12):DC05-8.

3. Menezes CB, Frasson AP, Tasca T. Trichomoniasis - are we giving the deserved attention to the most common non-viral sexually transmitted disease worldwide? Microb Cell. 2016 Sep 5;3(9):404-19.

4. Sood S, Kapil A. An update on Trichomonas vaginalis. Indian J Sex Transm Dis. 2008;29(1):7-14.

5. Schwebke JR, Burgess D. Trichomoniasis. Clin Microbiol Rev. 2004 Oct;17(4):794-803.

6. Preethi V, Mandal J, Halder A, Parija SC. Trichomoniasis: An update. Tropical Parasitology. 2011;1(2):73-75.
7. Nassef NA, Afif AF, Bauni AA, Abo El-Nasr MF, Atia AF Evaluation of microscopy and polymerase chain reaction for diagnosis of symptomatic and asymptomatic female trichomoniasis. 2014;7(1):37-46.

8. McClelland RS, Sangare L, Hassan WM, Lavreys L, Mandaliya K, Kiarie J, Ndinya-Achola J, Jaoko W, Baeten JM. Infection with Trichomonas vaginalis increases the risk of HIV-1 acquisition. J Infect Dis. 2007 Mar 1;195(5):698-702.

9. Karaman U, Karadag N, Atambay NB, Kaya A, Daldal NU. A comparison of Cytological and Parasitological Methods in the Diagnosis of Trichomonas vaginalis. Turkiye Parazitoloji Dergisi. 2008;32(4):309-12.

10. Klinger EV, Kapiga SH, Sam NE, Aboud S, Chen CY, Ballard $\mathrm{RC}$, Larsen U. A Community-based study of risk factors for Trichomonas vaginalis infection among women and their male partners in Moshi urban district, northern Tanzania. Sex Transm Dis. 2006 Dec;33(12):712-8.

11. Rassjo EB, Kambugu F, Tumwesigye MN, Tenywa T, Darj E. Prevalence of sexually transmitted infections among adolescents in Kampala, Uganda, and theoretical models for improving syndromic management. J Adolesc Health. 2006 Mar;38(3):213-21.

12. Menezes CB, Mello MS, Tasca T. Comparison of permanent staining methods for the laboratory diagnosis of Trichomoniasis. REV Inst Med Trop Sao Paulo. 2016; 58:5.

13. Khamees SS. Prevalence of Trichomonas vaginalis among women in Albatnan District. Int. J. of Pharm. \& Life Sci. 2012 Dec;3(12):2177-80.

14. Radonjic IV, Dzamic AM, Mitrovic SM, Arsic Arsenijevic VS, Popadic DM, Kranjcic Zec IF. Diagnosis of Trichomonas vaginalis infection: The sensitivities and specificities of microscopy, culture and PCR assay. Eur J Obstet Gynecol Repord Biol. 2006 May 1;126(1):116-20.

15. Hussein AH, Saleh MH, Nagaty IM, Ghieth KA, El-Azab NA. Prevalence, Clinical Criteria and Sociodemographic Predictors of Trichomonas vaginalis Infection in Suspected Egyptian Women, Using Direct Diagnostic Techniques. Iranian Journal of Parasitology. 2015;10(3):432-40.

*Corresponding author:

Neena Piyush Doshi, Dept of Pathology, GMERS Medical College, Gotri, Vadodara (India)- 390021

Phone: +91 02652398008

Email: neenapdoshi@gmail.com

Date of Submission : 06.07.2017

Date of

Financial or other Competing Interests: None.

Date of Publication : 22.12.2017 